\title{
ABS 3D printed solutions for cryogenic applications
}

\section{E. Bartolomé ${ }^{1}$, B. Bozzo ${ }^{2}$, P. Sevilla ${ }^{1}$, O. Martínez-Pasarell ${ }^{3}$, T. Puig ${ }^{2}$, X. Granados ${ }^{2}$}

\author{
${ }^{1}$ Escola Universitària Salesiana de Sarrià (EUSS), Passeig Sant Joan Bosco, 74, 08017, Barcelona, Spain \\ ${ }^{2}$ Institut de Ciència de Materials de Barcelona (ICMAB-CSIC), Campus Universitat Autònoma de Barcelona, 08193 \\ Bellaterra, Spain \\ ${ }^{3}$ Laboratori de Seminologia i Embriologia, Fundació Puigvert, Barcelona, Spain
}

\begin{abstract}
3D printing has become a common, inexpensive and rapid prototyping technique, enabling the ad hoc fabrication of complex shapes. In this paper, we demonstrate that 3D printed objects in ABS can be used at cryogenic temperatures, offering flexible solutions in different fields. Firstly, a thermo-mechanical characterization of ABS 3D printed specimens at $77 \mathrm{~K}$ is reported, which allowed us to delimit the type of cryogenic uses where 3D printed pieces may be implemented. Secondly, we present three different examples where ABS 3D printed objects working at low temperatures have provided specific solutions: (i) SQUID inserts for angular magnetometry (low temperature material characterization field); (ii) a cage support for a metamaterial "magnetic concentrator" (superconductivity application), and (iii) dedicated tools for cryopreservation in assisted reproductive techniques (medicine field).
\end{abstract}

\section{Introduction}

Rapid prototyping techniques (Stereolithography, Selective Laser Sintering, Laminated Object Manufacturing, 3D printing...) allow the quick, layer-by-layer fabrication of 3D solid objects, starting from a digital archive [1]. These processes are ideal to build up unique models, complexshaped pieces or parts in relatively small numbers. Today, 3D printing [2] has become an incredibly popular technique, due to its simplicity and favorable price/performance ratio. A plethora of off the shelf $3 \mathrm{D}$ printers have recently come to market [3], with prices falling dramatically since 2010, making 3D printing an affordable technology for industrial [4], scientific [5], medical [6], educational [7] and even domestic use [8].

In the most common 3D printing process, the building material, provided in the form of a roll of thermoplastic wire, is extruded through a nozzle moving in the $x y$-plane, and the subsequent deposition of layers forms the object in 3 dimensions. Some 3D printers use an additional, supporting material, which must be removed from the object after fabrication. There are many different building materials available for 3D printing (nylon, resins, steel, chocolate...[9]), but most desk-top 3D printers use ABS (Acrylonitrile Butadiene Styrene). ABS is a thermoplastic with a low glass transition temperature $\left(T_{\mathrm{G}} \approx 105{ }^{\circ} \mathrm{C}\right)$ that solidifies quickly, and presents medium strength and performance at reasonable prices.

To our knowledge, the application of 3D printing in cryogenic-related areas has not been explored yet. At present, the cryogenic use of polymer materials is receiving growing attention, with a view to its application in space, superconducting, electronic, energy and biomedical areas.
The cryogenic mechanical properties (including shear strength, impact strength, fracture toughness, creep, slip, etc.) of polymers play a critical role in determining their applicability at low temperatures, for example, liquid nitrogen $(77 \mathrm{~K})$ or liquid helium $(4.2 \mathrm{~K})$.. Generally speaking, the cryogenic mechanical properties of polymers are low compared to metals. Nonetheless, they are finding their place as electrical and thermal insulators, non-magnetic supports, vacuum sealants and matrix materials for composites used in cryogenic environments [10].

Works on the characterization of thermo-mechanical properties of polymer materials at cryogenic temperatures have been recently reviewed by $\mathrm{S}$. $-\mathrm{Y}$. Fu [11]. The cryogenic tensile behavior of some polymers with good mechanical behavior, aromatic polyimide (Upilex-R, UpilexS, Kapton) semicrystalline (PEN, PPS, PPT) films and epoxy resins (with various reinforcement modifiers such as carbon nanotubes or MMT) has been reported. Adhesive resins with higher shear strengths for cryogenic uses are also being investigated [12], [13]. Since the brittleness of polymers may limit their use in cryogenic environments, the increase of the impact strength and fracture toughness by addition of different modifiers in polymers such as epoxy resins is being studied [14]. Thermal conductivity also plays a significant role in the cooling and heating of cryogenic systems. The thermal conductivity of nonconductive polymers is low, when compared to metals or ceramics, and a further decrease of this property is sought by addition of silica to resins [15].

ABS cryogenic performance has so far not been investigated, and most providers give $-20^{\circ} \mathrm{C}$ as the lower working temperature [16]. Certainly, as for other thermoplastics, the mechanical properties of ABS are expected to decrease at very low temperatures [17], [10]. 
Nevertheless, this disadvantage may be surpassed by the convenience of making rapid prototypes by 3D printing. Scientists working in cryogenic-related areas, such as superconductivity or biomedicine, often encounter the need to make unique, complex-shaped objects for their experimental set ups.

In this paper, we demonstrate that ABS 3D printed objects can be used at cryogenic temperatures, offering flexible solutions in different fields. In the first part of the paper we report a thermo-mechanical characterization (tensile tests and thermal compression) of 3D printed ABS probes at $77 \mathrm{~K}$. These tests served to delimit the maximum permitted stresses and, therefore, the type of applications where 3D printed objects may be used safely. In the second part, we present three different examples of 3D printed pieces that provided solutions for different cryogenic applications: They were conceived to: (i) tune a part on an already existing instrument (fabrication of SQUID insets for angular magnetometry measurements); (ii) provide non-magnetic mechanical support for a completely new superconducting set up (a "magnetic concentrator"); or (iii) enable the fabrication of dedicated, low temperature tools (instruments for in vitro insemination).

\section{Experimental details}

An HP uPrint SE office 3D printer [18] with a 203 x 152 x $152 \mathrm{~mm}$ chamber was used. The 3D printed objects were made in ivory ABSplus-P430 from Stratasys [19] modeling material. Its thermal, electrical and mechanical properties (at room temperature) are found in the datasheet [20]. This 3D printer model uses SR-30 thermoplastic as support material, which is wavewashed in a cleaning system using an active oxygen detergent after the fabrication. The objects were printed using the default "Solid" built filling style, with raster orientation $\left[45^{\circ} / 45^{\circ}\right]$ and zero air-gap, as defined in HP 3D printer, using the minimum available nominal layer thickness, $0.254 \mathrm{~mm}$ for optimal part finishing. It is noted that in practice, the top- and bottom-most layers are thicker $(0.303$ $\mathrm{mm})$ than intermediate layers.

Fracture morphology of the tensile tested samples was evaluated with a Jeol JSM 6400 electron microscope. Fracture surfaces were previously coated with a goldplatinum layer by sputter coating with a Balzers SCD 004 coater in order to maximize sample conductivity.

SQUID magnetometry measurements in the range of temperatures from $5-300 \mathrm{~K}$, and magnetic fields between $0-3$ $\mathrm{T}$ were performed in a commercial MPMS XL Quantum Design set up.

\section{Thermo-mechanical characterization}

The mechanical properties of ABS material at cryogenic temperatures have not been reported so far. Therefore, an initial mechanical characterization at $77 \mathrm{~K}$ was performed as an outlook, in order to probe and delimit the prospects of using ABS 3D-printed parts in cryogenic applications.

Some initial tensile tests were performed on a $500 \mathrm{~N}$ capacity laboratory-made testing machine under displacement control, originally developed to test the mechanical properties of superconducting coated conductors (CCs) [23], see Fig. 1a. The tests were performed at $T=77 \mathrm{~K}$, with the specimens immersed in liquid nitrogen at atmospheric pressure. Load $(F)$ and displacement $(\Delta L)$ were continuously measured throughout each test. The load was applied at a rate of $50 \mathrm{~g} / \mathrm{s}$. Young's modulus $(E)$ was calculated as the slope of the stress-strain (SS) relationship between 0.0025 and 0.005 strain, according to ref. [24], while the yield strength is given as the stress corresponding to the cutting between the SScurve and the straight line parallel to the tangent in the origin to the SS-curve, with $0.2 \%$ abscise $\left(\sigma_{0.2}\right)$.

Axial strains were measured using electrical resistance
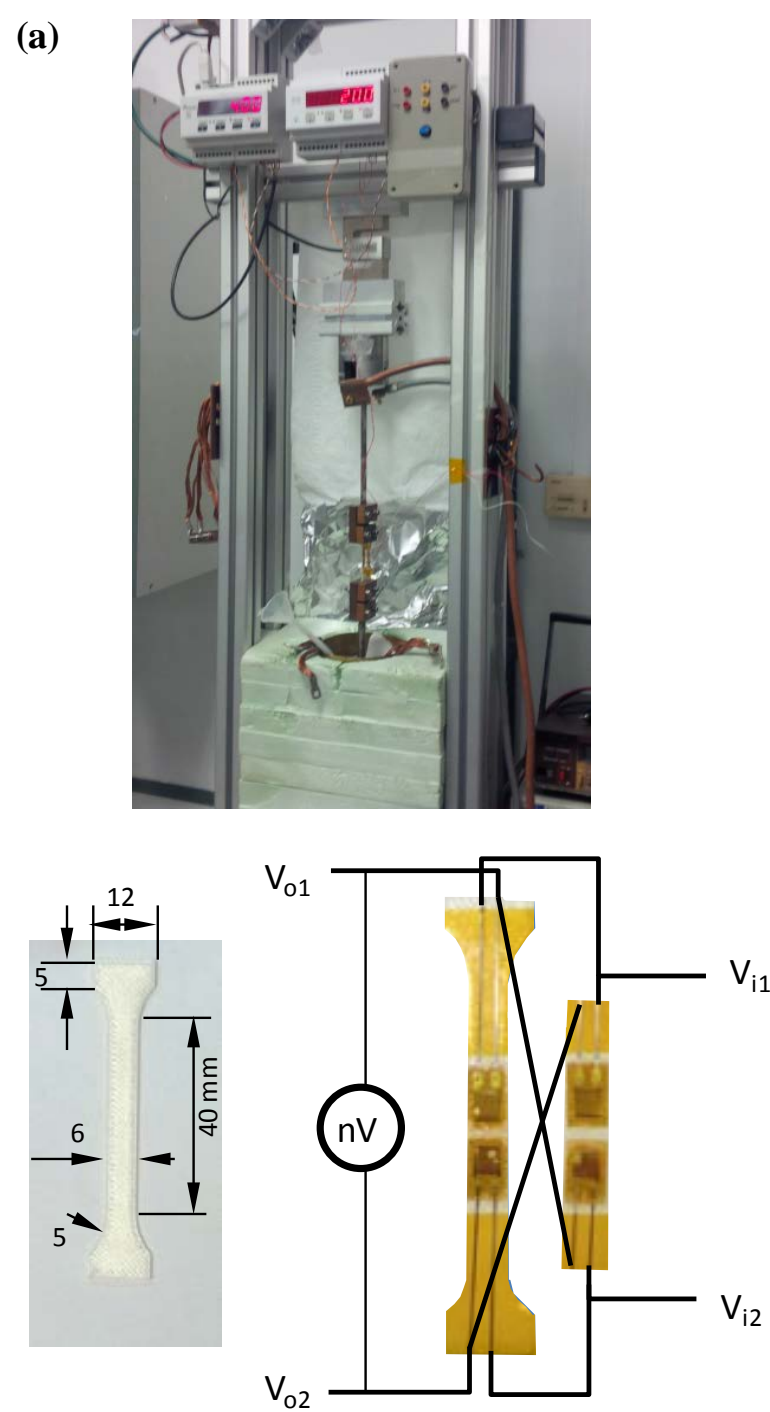

(b)

(c)

Fig. 1. (a) Tensile machine for measurements at $77 \mathrm{~K}$; (b) Shape and dimensions of 3D printed specimen for tensile tests. The measured thickness of the 3-layered "anisotropic xy" sample was $0.86 \mathrm{~mm}$. The raster angle was $45^{\circ}$ with respect to the specimen longitudinal angle and relative criss-cross between successive layers was $\left[45^{\circ} / 45^{\circ}\right]$; (c) Wheatstone configuration of strain gauges used to measure tensile stresses at cryogenic temperatures (left: sample specimen, right: reference sample). 
strain gauges (1LC-11-3/120) that were fixed on both sides of the center of the specimen, using epoxy glue, cured at $70^{\circ} \mathrm{C}$. A Wheatstone bridge configuration was adopted, using a second, reference sample next to the test specimen (Fig. 1c), in order to compensate the thermal contribution of RT-tobath cables.

The geometry and dimensions of the tensile test specimens are shown in Fig. 1b. Note that ASTM D 638 recommended dimensions could not be used because the jaws of the testing machine, designed initially to test CCs, allowed only $<8$ mmwide samples to be clamped. The specimen was 3D printed with the dogbone-shape lying in the $x y$-plane, and its thickness was $0.86 \mathrm{~mm}$, corresponding to three layers of printed material. One of the 3D printed specimens was heated above $T_{\mathrm{G}}$ so that the material softened and resolidified isotropically. Tests on this probe allowed us to compare the behavior of the 3D printed, anisotropic probe, with an isotropic one.

Figure 2 shows the stress $(\sigma)$ vs. strain $(\varepsilon)$ curves at $77 \mathrm{~K}$ measured for the two ABS studied specimens. The calculated Young's modulus $(E)$, yield strength $\left(\sigma_{0.2}\right)$, elongation at fracture $\left(\varepsilon_{F}\right)$ and ultimate tensile strength $\left(\sigma_{F}\right)$ for the two samples are summarized in Table 1 . The ultimate tensile stress for the isotropic sample $\left(\sigma_{\mathrm{F}}=16.1 \mathrm{MPa}\right)$ and elongation at fracture $\left(\varepsilon_{\mathrm{F}}=24.0 \%\right)$ are approximately an order of magnitude larger than for the anisotropic sample, which exhibits completely elastic behavior and fractures before plastic deformation appears. For a comparison, the elongation at break of other reported polymers investigated at $77 \mathrm{~K}$ was $11.9 \%$ (Upilex-S), 19.4\% (Kapton), 84.8\% (UpilexR), 8-12\% (PEN, 44-29\% crystallinity), 12.2\% (PPS film) [25], and 2.2-3.2\% (epoxy with 0-2\% CNT) [26].

Also, the Young's modulus of the 3D printed anisotropic sample is clearly lower. These inferior mechanical properties are a consequence of the poor interfacial adhesion between the constituent filaments and layers and the presence of sharp-edged pores which facilitate fracture propagation [27], [28].

Indeed, SEM images were taken on the fractured surfaces of the two samples at different magnifications (Figure 3). The micrographs revealed a completely brittle appearance on both samples, something to be expected since the testing temperature $(77 \mathrm{~K})$ was well below the glass transition temperature of the polymer. In addition, clear differences in the porosity of the two samples were observed. The

\begin{tabular}{|l|r|r|}
\hline & Isotropic & Anisotropic \\
\hline$E(\mathrm{MPa})$ & $305.3 \pm 0.5$ & $13.9 \pm 0.5$ \\
\hline$\sigma_{0.2}(\mathrm{MPa})$ & $3,6 \pm 0.1$ & -- \\
\hline$\sigma_{\mathrm{F}}(\mathrm{MPa})$ & $16,1 \pm 0.1$ & $0,63 \pm 0.1$ \\
\hline $\boldsymbol{\varepsilon}_{\mathrm{F}}(\%)$ & $24.0 \pm 0.2$ & $4.2 \pm 0.2$ \\
\hline
\end{tabular}

Table 1. Mechanical properties determined from tensile tests at $77 \mathrm{~K}$ for an isotropic ABS sample and a 3D printed, anisotropic sample.
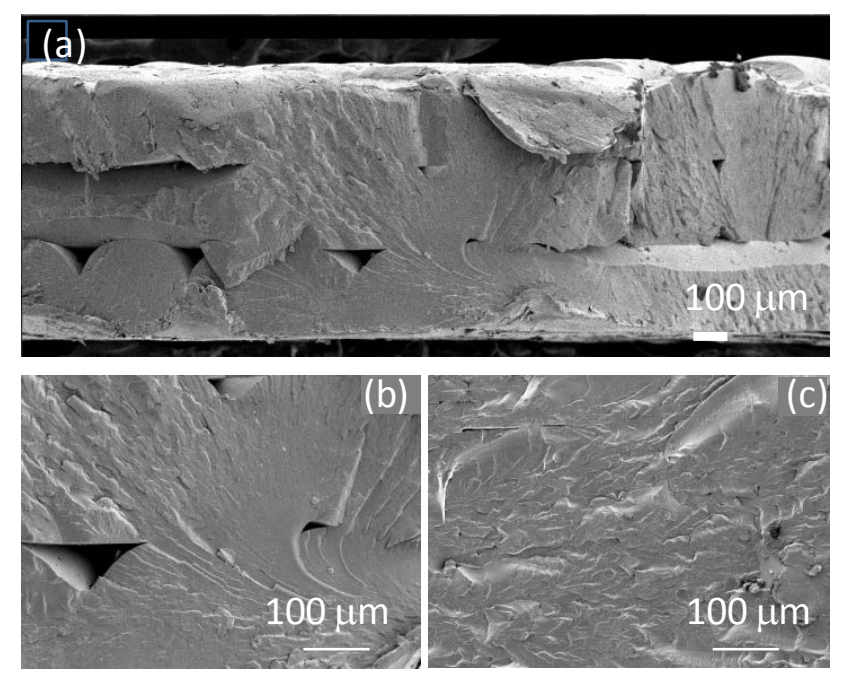

Fig. 3. SEM fracture micrographs of the tensile tested samples: a) General view of a fracture on the anisotropic sample; b) detail of the inter-wire space in the anisotropic sample; c) surface of fracture on the isotropic sample.

anisotropic sample showed sharply edged pores due to the layer by layer fabrication method, while the isotropic sample exhibited a lower porosity with rounded pores. These structural differences correlate well with the tensile test results, as sharp edged porosity generates a high stress concentration and is prone to provoke crack initiation [29], [30].

On the other hand, the thermal contraction of the anisotropic probe between room temperature and $77 \mathrm{~K}$ was determined by measuring the width of the specimen with a digital caliper as it was introduced into a liquid nitrogen bath. The measured strain was $\varepsilon=\Delta w / w=-2.39 \%$ and the estimated integral thermal expansion coefficient $\alpha=\varepsilon / \Delta T=$ $1.1 \times 10^{-4} \mathrm{~m} / \mathrm{m} / \mathrm{K}$.

It should be mentioned that a considerable number of works have appeared in recent years [21,22,31-34] showing that the mechanical performance at room temperature, finish quality and processing time of 3D-printed parts are significantly affected, in a non-trivial-manner, by a large number of different processing parameters, including e.g: the specific building material, specimen shape, build orientation, layer thickness, air gap between raster paths, raster angle and width, contour, build style etc... The systematic investigation of the individual and combined effect of the all these process parameters on the mechanical properties at $77 \mathrm{~K}$ would require a huge number of tests and is out of the scope of this outlook work. Future investigation using Design of Experiments (DOE) methods should allow establishing the influence of different processing parameters on the mechanic performance at cryogenic temperatures.

\section{ABS 3D printing for cryogenic applications}




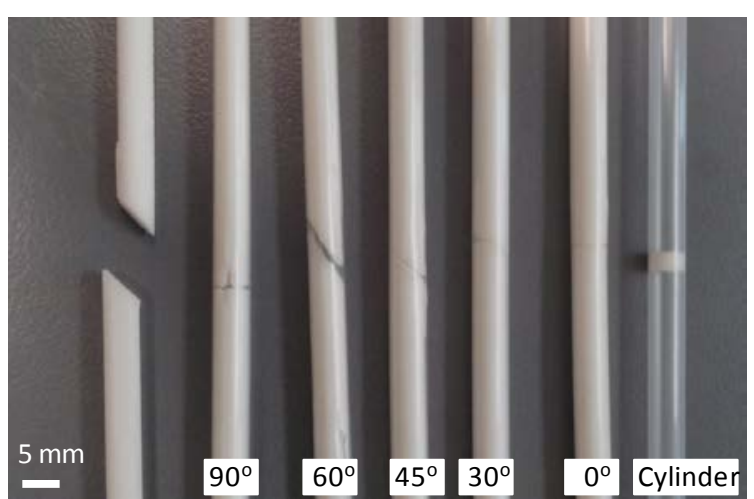

Fig. 4. 3D printed insets for SQUID angular magnetometry at $\theta=0^{\circ}, 30^{\circ}, 45^{\circ}$ and $90^{\circ}$ (a mounted sample is shown in the $60^{\circ}$ holder) and ABS printed cylinder used for its magnetic ,characterization.

for comparison.

The results of the prospect mechanical tests presented in the previous section demonstrate that $3 \mathrm{D}$ printed objects with standard ABS wires are not adequate for cryogenics applications under conditions of demanding stresses. Optimum benefit from 3D printed prototypes is instead foreseen for cryogenic applications requiring customized, special shapes and/or non-magnetic supports. We illustrate this idea with three different case examples.

\subsection{SQUID insets for angular magnetometry}

SQUID magnetometry is a standard technique used to characterize the magnetic properties of materials at temperatures down to $1.8 \mathrm{~K}$. Commercial SQUID magnetometers can be acquired with different types of insets, allowing bulk materials, platelets or powders to be lodged.

For instance, the typical characterization of superconducting (SC) thin-films includes the measurement by SQUID of the magnetization cycle as a function of the applied magnetic field, $M(H)$, and the temperature dependence of the magnetization, $M(T)$, in field-cool (FC) and zero-field cooled conditions (ZFC), with the magnetic field applied perpendicular to the thin-film ( $H$ parallel to the c-axis crystallographic direction). More sophisticated magnetization measurements under magnetic fields applied at different angles are used to study vortex pinning by different types of defects in YBCO type II SC films. These measurements require a devoted inset with a rotometer, whose non-negligible signal has to be carefully subtracted to deduce the sample signal.

As a practical alternative to make angular magnetometry, we proposed to fabricate special SQUIDs insets by 3D printing to orient the sample at certain fixed angles with respect to the magnetic field. This approach can be useful to test different samples under exactly the same angular conditions, or to evaluate the information that may be obtained from this type of measurements, before acquiring an expensive rotometer. The diamagnetic signal of the ABS, as we shall see, is very small compared to that of SC samples, so that its contribution can be ignored in most of the cases.

Figure 4 shows several 3D printed pieces, designed to fit inside a commercial SQUID cane (with a diameter of $5 \mathrm{~mm}$ ),

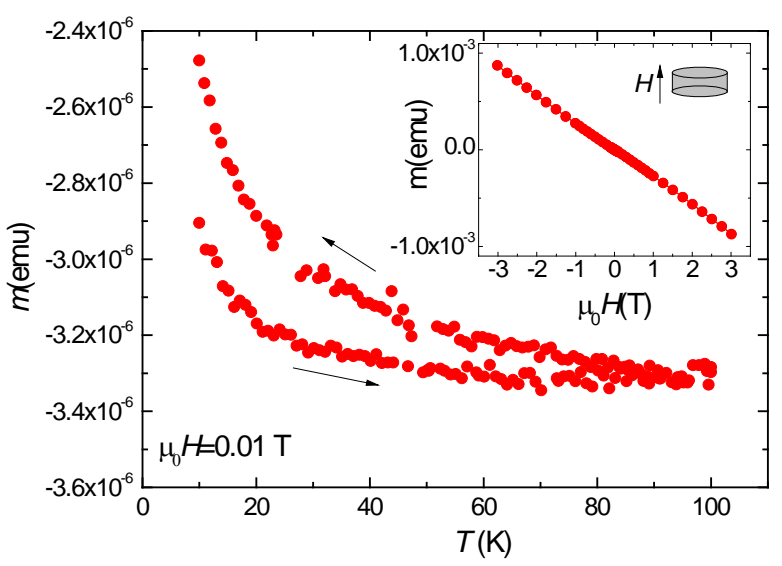

Fig. 5. Magnetic moment as a function of temperature at $0.01 \mathrm{~T}$ and magnetic moment as a function of the applied magnetic field at $5 \mathrm{~K}$ measured for a $3 \mathrm{D}$ printed $\mathrm{ABS}$ cylinder (5 mm diameter, $3 \mathrm{~mm}$ height).

which can hold SC thin-films at different angles $\left(\theta=0^{\circ}, 30^{\circ}\right.$, $45^{\circ}, 60^{\circ}$ and $90^{\circ}$ ).

A small cylinder (5 $\mathrm{mm}$ diameter, $3 \mathrm{~mm}$ height) was fabricated by 3D printing and characterized by SQUID in addition, to determine initially the magnetic response of the ABS material used (Figure 4, rightmost). Its magnetic moment as a function of the field, $m(H)$, measured at $5 \mathrm{~K}$ exhibits diamagnetic behavior, typical of organic aromatic plastics. The $m(T)$ measured at $0.01 \mathrm{~T}$ shows a decreasing behavior, probably due to the presence of some paramagnetic impurities. Additionally, some thermal hysteresis is observed during the $5 \mathrm{~K} \rightarrow 100 \mathrm{~K} \rightarrow 5 \mathrm{~K}$ measurement (Figure 5 ).
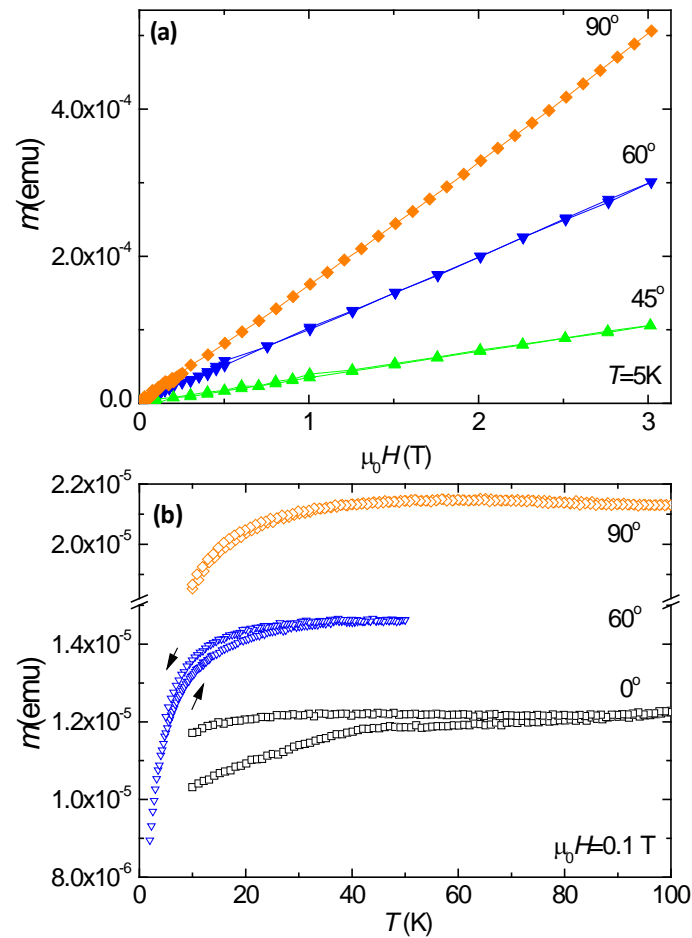

Fig. 6. (a) Magnetic moment as a function of the field at 5 $\mathrm{K}$, and (b) magnetic moment as a function of temperature at $0.1 \mathrm{~T}$ for the empty ABS insets with indicated angles. 

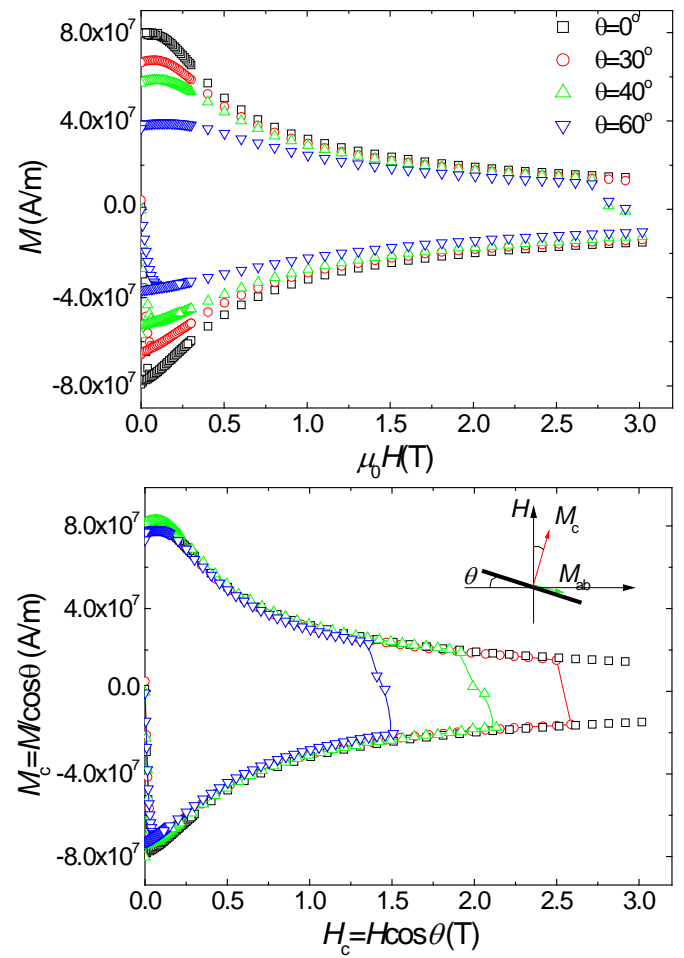

Fig. 7. (a) Magnetization cycle at $5 \mathrm{~K}$ of the YBCO-BZO thin-film measured with the magnetic field applied at $\theta=0$, 30,45 and $60^{\circ}$ with respect to the SC c-axis; b) all the data collapse when scaled as $M / \cos \theta$ vs. $H \cos \theta$.

In order to evaluate the magnetic contribution of the 3D printed holders, we measured the magnetic moment of all the empty insets (i.e. without sample inside). The fielddependence $m(H)$ at $5 \mathrm{~K}$ and temperature dependence $m(T)$ at $0.1 \mathrm{~T}$ of several angular-insets are shown in Figure 6. Note that, qualitatively, the $m(T)$ and $m(H)$ curves of the empty insets exhibit the inverse shapes of the curves measured for the ABS cylinder. This can be understood by considering that the SQUID magnetometer used for measurements is based on an extraction method. The magnetic moment is determined by measuring the currents generated at the gradiometer, caused by a material homogeneity break. When measuring the ABS cylinder, this symmetry break consists of airmaterial-air, whereas for the empty insert, it consists of material-air-material. We observe, on the other hand, that the magnetic signal of the ABS insets is negligible when compared with the magnetic moment typically found in SC thin-films (e.g. at $5 \mathrm{~K}$, the moment of the $0^{\circ}$-ABS inset is $m=1.1 \times 10^{-5}$ emu, while the SC film signal amounts 1.3 emu).

These insets were used to characterize a 2 x $3.5 \mathrm{~mm}^{2}, 250$ $\mathrm{nm}$ thickness superconducting YBCO-BZO nanocomposite thin-film grown on a LAO substrate [35].

Magnetic hysteresis cycles $M(H)$ measured at $5 \mathrm{~K}$, with the sample at different orientations $\left(\theta=0,30,45,60^{\circ}\right)$ with respect to the applied magnetic field are shown in Figure 7a. Our SQUID magnetometer is prepared to measure only the longitudinal component of the magnetization, $M_{\mathrm{L}}$, parallel to

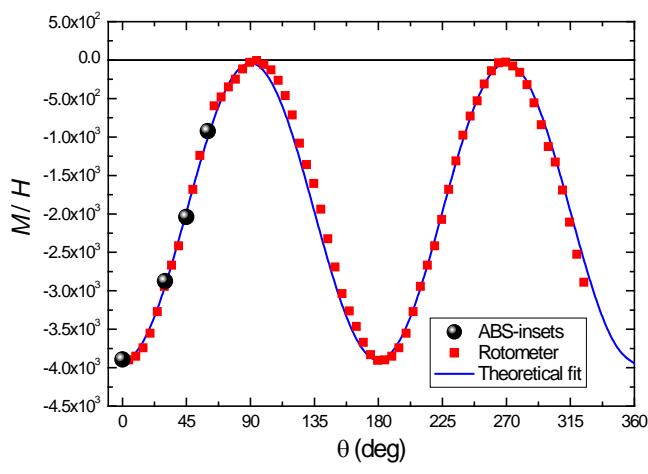

Fig. 8. Angle dependence of the longitudinal magnetization divided by the applied field $\left(H=10 \mathrm{Oe}<H_{\mathrm{c} 1}\right)$ in the Meissner state. Experimental data measured using the ABS-angleinsets $(\bullet)$, a SQUID rotometer (ם), and theoretical fit to Eq. [2], (line).

the vertically applied magnetic field. For thin SC films with extreme large aspect ratio $(a / t)$, under fields inclined at angles $\theta<\theta_{\mathrm{c}}=\tan ^{-1}(a / t) \approx 89.9^{\circ}$, and low temperatures $(T<<$ critical temperature $T_{\mathrm{c}}$ ), it has been demonstrated that the magnetization is $c$-axis locked, so that $M_{\mathrm{L}}$ can be approximated as [36]:

$$
M_{L}=M_{c} \cos \theta-M_{a b} \sin \theta \approx M_{c} \cos \theta
$$

Thus, the magnetization data measured at different orientations, scaled as $M_{\mathrm{c}} \approx M_{L} / \cos \theta$, and represented as a function of the $c$-axis of the magnetic field $\left(H_{\mathrm{c}}=H \cos \theta\right)$ are expected to fall under a universal curve. Figure $7 \mathrm{~b}$ shows that this is indeed the case in our measurements, showing that the SC film inclinations were well determined by the ABS insets.

On the other hand, in the Meissner state of the SC film, at sufficiently low fields $\left(H<H_{\mathrm{c} 1}\right)$, the theoretical dependence of the $M_{\mathrm{L}}$ component as a function of the angle $\theta$ is expected to follow the dependence [37]:

$$
-\frac{M_{L}}{H}=\frac{1}{2 v} \cos ^{2} \theta+\frac{1}{1-v} \sin ^{2} \theta,
$$

where $v$ is the demagnetization factor of the sample, basically given by the thickness $t$ of the film divided by its width $a$. Figure 8 shows that the $M / H(\theta)$ dependence experimentally determined in Meissner conditions could be indeed nicely fitted to this theoretical expression. Moreover, the data recorded using the 3D printed insets lies on top of the $M / H(\theta)$ data recorded using a rotometer.

In conclusion, the above consistency tests demonstrate that the SC samples could be correctly orientated using the ABS 3D printed inserts with approximately $1^{\circ}$ of accuracy.

\subsection{Magnetic concentrator support}

The theoretical concept of a superconductor (SC)ferromagnetic (FM) metamaterial, shell allowing the concentration of magnetic energy was proposed in 2012 


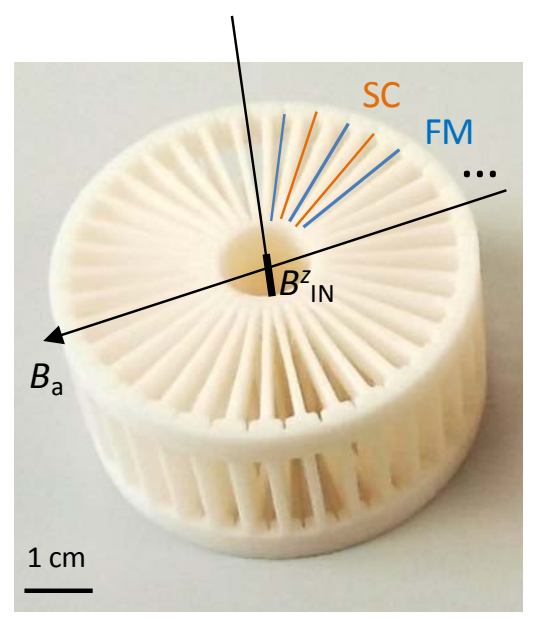

Fig. 9. $3 D$ printed support in ABS fabricated for the experimental realization of a "magnetic concentrator", working at $77 \mathrm{~K}$.

[38],[39]. The actual achievement of this "magnetic concentrator" device required the precise radial alignment of several superconducting (SC) and ferromagnetic (FC) lamellae into a non-magnetic support, to be placed into a liquid nitrogen bath.

Figure 9 shows an overall view of the cage-like support that was designed and fabricated in ABS by 3D printing for this application. The cylindrical support, with an external radius of $31 \mathrm{~mm}$, and height of $33 \mathrm{~mm}$, was hollowed out in the center (inner radius of $6.5 \mathrm{~mm}$ ) in order to lodge a magnetic Hall probe sensor. The support included 36 homogeneously distributed slots, to alternately place 18 SC and 18 FM 22 x $30 \mathrm{~mm}$ rectangular pieces. The support was fabricated with openings in the lateral face and interior walls to ensure the free circulation of the liquid nitrogen. The object was 3D printed with the axis of rotation perpendicular to the deposition $x y$-plane. Thorough washing of the piece in an active oxygen bath was required to remove the remaining support material.

The complex-shape of this cage-like support would have been very expensive to produce by conventional methods. Moreover, the accurate radial placement of the SC and FC lamellae was essential to test the magnetic concentration effect, and this would have been difficult by other means. In this example the 3D printed object worked simply as a geometric support, and thus was not subjected to important stresses. The support was thermally cycled between room temperature and $77 \mathrm{~K}$ about 8 times without breaking, allowing different magnetic concentration experiments to be carried out. It was demonstrated that the magnetic field in the center of the metamaterial shell $\left(B_{\mathrm{IN}}{ }^{\mathrm{z}}\right)$ was increased by a factor of 2.7 with respect to the external field $\left(B_{\mathrm{a}}\right)$ applied perpendicularly to the shell axis, in excellent agreement with 3D numerical simulation predictions [40].

\subsection{Tools for assisted reproductive techniques}

Since the middle of the $20^{\text {th }}$ century, cryopreservation techniques for human spermatozoa, oocytes, and embryos [41-44] have been developed and introduced routinely in assisted reproductive laboratories. In this section we show how 3D printed pieces were used to facilitate the achievement of two different steps in the cryopreservation protocol at the in vitro laboratory of the Puigvert Fundation (PF) in Barcelona.

Vitrification is nowadays the preferred cryopreservation strategy for oocytes and embryos because it is simple, it has a good level of successful survival rates and good clinical results. This method consists of transforming cells in a glassy state inside and outside of the cell. The oocytes/embryos are equilibrated in a cryoprotectant solution, loaded onto a holder tool with minimal volume of the solution containing the oocytes/embryos and then directly plunged in liquid nitrogen. The ultra-rapid cooling step avoids ice-crystal formation. As a holder tool, it is common to use a thin film strip attached to a hard plastic holder (e.g. at F. Puigvert laboratory, a $0.4 \mathrm{~mm}$ wide, $20 \mathrm{~mm}$ long, $0.1 \mathrm{~mm}$ thick Cryotop ${ }^{\circledR}$ from Kitazato, Japan [45] is used). To protect the oocytes/embryos from mechanical damage during storage, the film must be introduced into a long, narrow plastic tube cap. This delicate
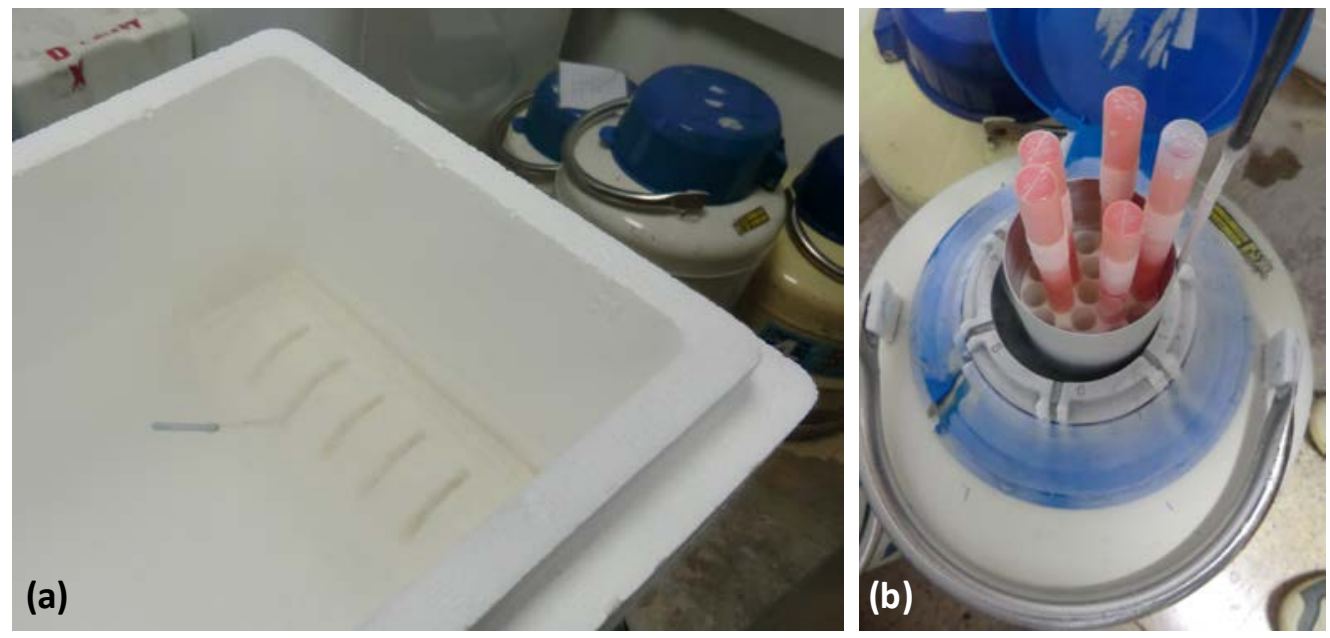

Fig. 10. (a) 3D printed support block for five straws immersed in liquid nitrogen; (b) 3D printed goblet holder inside a canister, for cryopreservation of oocytes/embryos in a LN vessel. 
operation must be done while keeping the straw permanently immersed in liquid nitrogen (LN). Thus, the LN container must be large enough to allow correct manipulation, and thermally isolated stainless-steel or polystyrene vessels are often used for this step.

It would be ideal to have support devices to hold different straws correctly in place while manipulating a new one inside the LN. Recently, a commercial solution from KitazatoDibimed has appeared, consisting of an aluminum block to support two straws at fixed positions, fitting in a steel-box of the same housing [45]. However, this closed solution may not meet the requirements of every lab. By using a 3D printer, we designed and manufactured an adapted solution fulfilling the specific requirements of the PF lab: a straw stocker, exactly fitting into the available polystyrene vessel, prepared to hold up to 5 straws in a row, at two different angular positions, vertically $\left(90^{\circ}\right)$ and inclined $\left(60^{\circ}\right)$, (Fig. 10a).

In a subsequent step, the vitrified oocytes/embryos are stored in cryogenic tanks, which are vacuum insulated vessels of aluminum with fiberglass neck construction. E.g., the bank of embryos (B2026, Cryo Diffusion, France) at FP has a capacity of $26 \mathrm{l}$ of liquid nitrogen, and can store up to 10 canisters (66 mm internal Ø), hanging from the vessel neck. Usually, all the cryotops of one patient are identified, grouped and introduced in a plastic goblet $(10 \mathrm{~mm} \varnothing / 117$ $\mathrm{mm}$ long) with a cap (11 mm Ø/550 mm long). The different goblets are then distributed in the canisters of the bank without any fixing system. This can be problematic, especially during manipulation of the canisters to insert or extract samples, the goblets are free to float in the bath, with the subsequent risk to lose a sample. No efficient commercial solution is available. To solve this problem, we designed and 3D printed a cylindrical support, fitting into the canister, with capacity to locate a maximum of 19 goblets (Figure 10b), which solved the issue of the floating goblets.

\section{Conclusions}

In this work we explored the use of ABS 3D printed objects for cryogenic applications. The tensile tests performed at 77 $\mathrm{K}$ in a 3 layer-thick 3D printed sample evidence that brittle fracture occurs under a stress of $0.61 \mathrm{MPa}$, a value an order of magnitude smaller than in an isotropic specimen of the same geometry. This brittle mechanical behavior at low temperatures, foreseeable in a thermoplastic material like ABS, would at first sight appear to count against the use of $3 \mathrm{D}$ printed pieces in applications where large stresses must be sustained. On the contrary, 3D printing is ideal for fabricating complex-shaped, non-magnetic supports or dedicated tools for different cryogenic applications. Indeed, we have shown three different examples where 3D printed objects (SQUID insets, a support for a magnetic concentrator, and in vitro tools) provided flexible solutions for experimentalists. This opens a large range of possibilities for the use of 3D printed objects in cryogenic environments in different fields, e.g. in superconductivity, energy, biomedicine etc.

\section{Acknowledgements}

We acknowledge financial support from Spanish Ministry of Economy and Competitiveness through the "Severo Ochoa" Programme for Centres of Excellence in R\&D (SEV-2015-
0496), CONSOLIDER Excellence Network (MAT201568994-REDC), COACHSUPENERGY project (MAT201451778-C2-1-R). The authors would also like to thank J. PratCamps from UAB Electromagnetism Group for his participation in the design of the concentrator support. M. Turner is acknowledged for English checking of the manuscript.

\section{References}

[1] Chua CK, Kua LF. Rapid prototyping: Principles and Applications in Manufacturing. Wiley; 1997.

[2] Crump SS. Apparatus and method for creating three-dimensional objects, 1992.

[3] www.3ders.org n.d.

[4] www.stratasys.com/industries n.d.

[5] Cesaretti G, Dini E, De Kestelier X, Colla V, Pambaguian L. Building components for an outpost on the Lunar soil by means of a novel 3D printing technology. Acta Astronaut 2014;93:430-50. doi:10.1016/j.actaastro.2013.07.034.

[6] Rengier F, Al. E. No Title. Int J CARS 2010;93:430.

[7]

www.educatorstechnology.com/2013/03/im portance-of-3d-printing-in-education.html n.d.

[8] Kelly H. Study: at home 3D printing could save consumer "thousands." CNN 2013.

[9] Blog.tinkercard.com/materialsguide; www.shapeways.commaterials n.d.

[10] Hartwig G. Polymer properties at room and cryogenic temperatures. New York: 
Springer Science+Business Media LLC; 1994.

[11] Kalia S, Fu SY. Polymers at cryogenic temperatures. vol. 9783642353. 2013. doi:10.1007/978-3-642-35335-2.

[12] Huang CJ, Fu SY, Zhang YH, Lauke B, Li LF, Ye L. Cryogenic properties of $\mathrm{SiO} 2 /$ epoxy nanocomposites. Cryogenics (Guildf) 2005;45:450-4. doi:10.1016/j.cryogenics.2005.03.003.

[13] Bardalen E, Akram MN, Malmbekk H, Ohlckers P. Review of Devices, Packaging, and Materials for Cryogenic Optoelectronics. J Microelectron Electron Packag 2015;12:189-204. doi:10.4071/imaps.485.

[14] Domun N, Hadavinia H, Zhang T, Sainsbury T, Liaghat GH, Vahid S. Improving the fracture toughness and the strength of epoxy using nanomaterials - a review of the current status. Nanoscale 2015;7:10294329. doi:10.1039/C5NR01354B.

[15] Kalaprasad G, Pradeep P, Mathew G, Pavithran C, Thomas S. Thermal conductivity and thermal diffusivity analyses of low-density polyethylene composites reinforced with sisal, glass and intimately mixed sisal/glass fibres. Compos Sci Technol 2000;60:2967-77. doi:10.1016/S0266-3538(00)00162-7.

[16]

htttp://www.dynalabcorp.com/technical_inf o_abs.asp; www.tangram.co.uk/TI-PolymerABS.html\#GP n.d.

[17] Perkins WG. Polymer toughness and impact resistance. Polym Eng Sci 1999;39:2445-60. doi:10.1002/pen.11632.
[18] htttp://www.hp3dprinting.co.uk/uprintse.htm n.d.

[19]

http://www.stratasys.com/materials/fdm/abs plus n.d.

[20]

http://www.stratasys.com/ /media/Main/Se cure/Material\%20Specs\%20MS/FortusMaterial-Specs/Fortus-MS-ABSplus-01-13web.pdf n.d.

[21] Mohamed O a., Masood SH, Bhowmik JL. Optimization of fused deposition modeling process parameters: a review of current research and future prospects. Adv Manuf 2015:42-53. doi:10.1007/s40436-014-00977.

[22] Zou R, Xia Y, Liu S, Hu P, Hou W, Hu Q, et al. No Title. Compos Part B 2016;99:50613.

[23] Konstantopoulou K, Sarazin M, Granados X, Pastor J., Obradors X. Effect of the axial stress and the magnetic field on the critical current and the electric resistance of the joints between HTS coated conductors. Supercond Sci Technol 2015;28:064001.

[24] Faes M, Ferraris E, Moens D. Influence of Inter-layer Cooling time on the Quasi-static Properties of ABS Components Produced via Fused Deposition Modelling. Procedia CIRP 2016;42:748-53. doi:10.1016/j.procir.2016.02.313.

[25] Yano O, Yamaoka H. Cryogenic properties of polymers. Prog Polym Sci 1995;20:585613. doi:10.1016/0079-6700(95)00003-X.

[26] Chen ZK, Yang JP, Ni QQ, Fu SY, Huang YG. Reinforcement of epoxy resins with multi-walled carbon nanotubes for 
enhancing cryogenic mechanical properties. Polymer (Guildf) 2009;50:4753-9. doi:10.1016/j.polymer.2009.08.001.

[27] Tekinalp HL, Kunc V, Velez-Garcia GM, Duty CE, Love LJ, Naskar AK, et al. Highly oriented carbon fiber-polymer composites via additive manufacturing. Compos Sci Technol 2014;105:144-50. doi:10.1016/j.compscitech.2014.10.009.

[28] Kar GP, Biswas S, Bose S. X-ray micro computed tomography, segmental relaxation and crystallization kinetics in interfacial stabilized co-continuous immiscible PVDF/ABS blends. Polymer (Guildf) 2016;101:291-304. doi:10.1016/j.polymer.2016.08.071.

[29] Introduction to Contact Mechanics. Spinger Science\&Business Media; 2007.

[30] Anderson TL. Fracture Mechanics: Fundamentals and Applications. Third Edit. 2005.

[31] Wang CC, Lin T-W, Hu S-S. Optimizing the rapid prototyping process by integrating the Taguchi method with the Gray relational analysis. Rapid Prototyp J 2007;13:304-15. doi:10.1108/13552540710824814.

[32] Ahn S, Montero M, Odell D, Roundy S, Wright PK. Anisotropic material properties of fused deposition modeling ABS. Rapid Prototyp J 2002;8:248-57. doi:10.1108/13552540210441166.

[33] Sood AK, Ohdar RK, Mahapatra SS. Parametric appraisal of mechanical property of fused deposition modelling processed parts. Mater Des 2010;31:287-95. doi:10.1016/j.matdes.2009.06.016. deposition modelling (fdm) process parameter prediction and optimization using group method for data handling (gmdh) and differential evolution (de). Int J Adv Manuf Technol 2014;73:509-19. doi:10.1007/s00170-014-5835-2.

[35] Obradors X, Puig T, Pomar A, Sandiumenge F, Mestres N, Coll M, et al. Progress towards all-chemical superconducting YBa2Cu3O7-coated conductors. Supercond Sci Technol 2006;19:S13-26. doi:Doi 10.1088/0953-2048/19/3/003.

[36] Zhukov AA, Perkins GK, Bugoslavsky Y V., Caplin AD. No Title. Phys Rev B 1997;56:2809.

[37] Candia S, Civale L. No Title. Supercond Sci Technol 1999;12:192.

[38] Navau C, Prat-Camps J, Sanchez A. Magnetic energy harvesting and concentration at a distance by transformation optics. Phys Rev Lett 2012;109.

doi:10.1103/PhysRevLett.109.263903.

[39] Prat-Camps J, Sanchez A, Navau C. Superconductor-ferromagnetic metamaterials for magnetic cloaking and concentration. Supercond Sci Technol 2013;26:074001. doi:10.1088/09532048/26/7/074001.

[40] Prat-Camps J, Navau C, Sanchez A. Experimental realization of magnetic energy concentration and transmission at a distance by metamaterials. Appl Phys Lett 2014;105. doi:10.1063/1.4903867.

[41] Bunge RG, Keettel WC, Sherman JK. Clinical use of frozen semen: report of four cases. Fertil Steril 1954;5:520-9. 
[42] Trunson A, Mohr L. Human pregnancy following cryopreservation, thawing and transfer of an eight-cell embryo. Nature 1983;305:707-7092.

[43] Pregnancy after human oocyte cryopreservation. Lancet 1986;1:884-6.

[44] Kuwayama M. Highly efficient vitrification for cryopreservation of human oocytes and embryos. Theriogenology 2007;67:73-80.

[45] http://www.kitazatodibimed.com/ver/206/Cryotop-SCAccessories.html n.d. 